Review Article

Open Access

\title{
Review on Immunological Biomarkers in Gliomas
}

\author{
Nagalakshmi CS and Santhosh NU \\ ${ }^{1}$ Department of Biochemistry, Akash Institute of Medical Sciences and Research centre, Devanahalli, Bangalore, Karnataka, India \\ ${ }^{2}$ Consultant Neurosurgeon, Aster CMI Hospital, Sahakarnagar, Bangalore North, Karnataka, India
}

\section{Article Info}

*Corresponding author:
Nagalakshmi CS
Associate Professor
Department of Biochemistry
Akash Institute of Medical Sciences and
Research centre
Devanahalli-562110
Bangalore, Karnataka
India
E-mail: nagu.smile@gmail.com

Received: March 25, 2019

Accepted: April 10, 2019

Published: April 18, 2019

Citation: Nagalakshmi CS, Santhosh NU. Review on Immunological Biomarkers in Gliomas. Madridge J Immunol. 2019; 3(2): 84-88.

doi: $10.18689 / \mathrm{mjim}-1000119$

Copyright: ( $\subset 2019$ The Author(s). This work is licensed under a Creative Commons Attribution 4.0 International License, which permits unrestricted use, distribution, and reproduction in any medium, provided the original work is properly cited.

Published by Madridge Publishers

\begin{abstract}
Gliomas are the most common subgroup of primary malignant brain tumors in adults, constituting $>40 \%$ of all primary CNS neoplasms. Although all gliomas originate from neuroepithelial tissues, they vary considerably in morphology, location, genetic alterations and in their response to therapy. The most malignant of gliomas (Grade IV) is Glioblastoma Multiforme (GBM), causing over 10,000 deaths each year in the US alone. Despite robust therapeutic advances, median survival for GBM still remains 14-20 months with very high tumor recurrence rate. Various investigation modalities are available for establishing diagnosis of glioma, like: CT scan, MRI, X-ray, spinal tap, angiogram, myelogram \& biopsy, though, histopathology represents the gold standard for their typing \& grading. However, even this remains unsatisfactory because of the lack of reproducibility and absence of precision. Development of objective, diagnostic, prognostic \& predictive markers for these lethal neoplasms is therefore a priority. Biomarkers for glioma can be identified in various biological samples like: DNA, mRNA, cell surface receptors, transcription factors, secretory proteins, metabolites or processes such as proliferation, angiogenesis or apoptosis. Tumor biomarkers help oncologists in managing gliomas at various levels, from screening till assessment of longitudinal response to therapy. Identifying the molecular \& pathogenetic characteristics of glioma regulation network may increase the precision of customized medication. Further, the proteomic approach has the potential to identify novel diagnostic, prognostic and therapeutic biomarkers. In the near future, improved proteomic profiling is anticipated to bring about a merger of biology, engineering and informatics, with a profound impact on glioma research and treatment. Optimization of experimental design and validation in independent cohorts, improved multiplex proteomic methodologies and bioinformatics tools, and their integration with genetic and metabolomic profiling technologies promise to play critical roles in the post proteomics era of cancer diagnosis and treatment.
\end{abstract}

Keywords: Glioma; Glioblastoma Multiforme; Brain Tumors; Biomarkers; Immune Markers; Immunotherapy.

\section{Introduction}

Brain tumors are widespread with an incidence of 12.8 per 100,000 population. Glioma, a devastating condition, originating from glial cells of brain or spine, is the most common subgroup of primary malignant brain tumors in adults, constituting $>40 \%$ of all primary CNS neoplasms [1-4]. Although all gliomas originate from neuroepithelial tissues, they vary considerably in morphology, location, genetic alterations and in their response to therapy. The World Health Organization has classified gliomas, based on histological tumor type into: (i) Astrocytic (ii) Oligodendroglial (iii) Mixed oligo-astrocytic \& (iv) Ependymal. They present in various grades of malignancy - I, II, III or IV. Grade IV, the most malignant of gliomas, is Glioblastoma Multiforme (GBM), causing over 10,000 deaths each year in the US alone [4]. 
Gliomas are not so easy to manage. Despite robust therapeutic advances, median survival for GBM, in this era of radiation \& temozolamide, is only $14-20$ months and tumor recurrence rate is quite alarming $[1,2,5]$.

\section{Need for Diagnostic Biomarkers for Glioma}

Gliomas are difficult to remove due to various reasons: (a) certain chemotherapeutic drugs cannot reach gliomas directly because of blood-brain barrier (b) gliomas can infiltrate tissues around them and so the traditional approaches of surgery, radiation and chemotherapy, cannot remove them completely, and finally (c) gliomas may be made up of more than one tumor cell type \& so, any particular chemotherapy drug, cannot kill all tumor cell types [1].

Currently, various investigation modalities are available for establishing diagnosis of glioma, like: CT scan, MRI, X-ray, spinal tap, angiogram, myelogram \& biopsy, though, histopathology represents the gold standard for their typing $\&$ grading. However, even this remains unsatisfactory because of the lack of reproducibility and absence of precision in terms of prognosis, as evidenced by large inter-observer variability $[1,4,6,7]$. Therefore, development of objective glioma biomarkers for diagnosis, prognosis and therapy are an urgent mission for the scientists in near future.

Biomarker is a characteristic that can be measured as an indicator of normal biological process, pathogenic process or a pharmacological response to a therapeutic intervention. And tumor biomarkers encompass a wide variety of objects like: DNA, mRNA, cell surface receptors, transcription factors, secretory proteins, metabolites or processes such as proliferation, angiogenesis or apoptosis. These markers are produced either by the tumor tissue itself or by other tissues, in response to tumor or its associated factors, like inflammation. Biomarkers for glioma can be identified in various biological samples such as: serum, cerebrospinal fluid, cyst fluid, glioma cell lines or from glioma tissues $[1,4]$.

Numerous ways by which tumor biomarkers can help oncologists include, but not limited to: profiling the cancer predisposition, its early diagnosis, state of cancer development, prognosis after treatment, deciding upon the drugs $\&$ dosages etc. They not only prove beneficial while screening the targeted drug for tumor treatment, but also provide unique information related to longitudinal response to therapy $[1,4]$.

As mentioned above, highly invasive nature of gliomas, without obvious boundaries, make its complete surgical resection hard to accomplish [5,8-11]. Chemotherapy and radiation target tumour cells in their growth phase but fail to affect the quiescent glioma stem cells. This is why invasive glioma has a low cure rate and a high recurrence rate. In this direction, a promising new tool to combat GBM is immunotherapy, which has emerged from intensive research, revealing that although highly immunogenic, it actively suppresses the host anti-tumoral immune response through a number of mechanisms $[2,12,13]$. A deeper understanding of specific molecular markers is the core part of such immunotherapy.

Recent work by Alexiou et al. contributes to the use of immune mediators as prognostic surrogates of GBM aggressiveness \& survival and may be clinical indicators, of which patients are likely to benefit in current and future immunotherapeutic trials $[2,14,15]$. In a study, Alexiou et al. $[2,10,14]$ demonstrated a lower neutrophil-to-lymphocyte ratio (NLR) as an independent prognostic marker of survival. Using a cutoff value of NLR $<4.7$, both overall survival (OS) and progression-free survival (PFS) were significantly longer. This study supports another work $[2,16]$ which also revealed NLR as an independent prognostic marker, using a cut-off value of 4 , and is consistent with other studies demonstrating the prognostic value of NLR in other tumor types $[2,17,18]$.

Extensive work has explored the role of tumorinfiltrating lymphocytes (TILs) in glioma, both as markers of tumor aggressiveness and with patient survival, showing that infiltration of cytotoxic CD8 + T lymphocytes are decreased and $\mathrm{CD} 4+$ and $\mathrm{CD} 4+/ \mathrm{CD} 25+/$ FoxP3 + (Treg) populations of $\mathrm{T}$ lymphocytes increased in higher grade tumors, with increased Treg infiltration indicating a worse prognosis $[2,19]$. Han et al. $[2,20]$ established that NLR reflects the presence of TILs within the tumor milieu.

In another study, Berghoff et al. [2,21], demonstrated that improved survival was not associated with TIL density (or PD-L1 overexpression), in contrast to other studies. Routine analysis of TILs is difficult in clinical settings and so it is important that the study of Alexiou et al. $[2,14,15]$ mirrors the use of serum kynurenine $\&$ tryptophan levels as another biomarker of the immunosuppressive effect of IDO (Indoleamine 2,3-dioxygenase 1) catabolism in gliomas, which demonstrated prognostic significance for overall survival [2,22].

Taken together, the work of Alexiou et al. [2,14], Bambury et al. [2,16] and Han et al. [2,20] elaborate upon the role of TILs in GBM via an easily obtainable, non-invasive, serum biomarker proxy. These papers reinforce and expand upon the paradigm that GBM is a lymphocyte-suppressing tumor and that lymphocyte down-regulation is by itself a marker of aggressive tumor pathology.

Another article by Alexiou et al. [2,15] in patients undergoing surgery for various intracranial tumors, revealed that lower serum IgE levels in GBM patients were a prognostic marker associated with poorer survival and higher-grade in gliomas. IgE levels in gliomas and meningiomas were also significantly lower than that in metastatic tumors. This supports prior work on $\lg E$ and allergies in the development of malignancies. Further, there was an inverse relationship between allergies and gliomas $[2,23]$. A number of previous studies also suggest that a general atopy phenotype rather than a specific allergen that is correlated with decreased glioma risk $[2,23,24] \&$ it is consistent with total serum IgE levels. Thus, it is not a limitation of Alexiou's study that allergen-specific IgE antibodies were not elucidated. 
GBM continues to be a catastrophic tumor, with eventual recurrence and poor survival, due to tumor-based immunosuppression. Immunotherapy is a promising addition to the current standard of care. The studies highlighted here $[2,14,15]$ emphasize the growing role of immune cells and antibodies in the understanding of both gliomagenesis, glioma progression, and the development of aggressive and treatment-resistant tumor types. They do not identify a causative role for inflammatory mediators as either protective or tumor-suppressive, but rather begin to pinpoint immune phenotypes of GBM. Furthermore, they provide easily measurable biomarkers for immunosuppressive GBM phenotypes, which are prognostic for patient survival and are clearly applicable to clinical trials for novel immunotherapies. Subsequent work is needed to correlate these findings with other prognostic GBM markers, its subtypes and imaging characteristics [2,25]. In fact, immunotherapy-induced changes on neuroimaging have been incorporated into the newest response assessment guidelines ("iRANO") $[2,26]$ with doubtful possibility that these serum biomarkers do correlate. It is equally important to identify mechanistic role of $\lg \mathrm{E}$, and also CD4+ Th2 cells, CD4+/CD25+/FoxP3+ Treg cells, or the IgE-driving cytokines IL-4 \& IL-13, in the glioma milieu and the promulgation of tumor immunosuppression. Of further value would be to explore upon these prognostic findings, to determine if the serum NLR or IgE levels are useful to assess treatment response [2].

There are few other markers related to the prognosis of patients with glioma. Ozawa et al. [5,27] found that NF1 deletions may render neuronal precursor tumors into mesenchymal subtypes. Increased expression of Myc has been associated with the grading of gliomas $[5,28]$. It plays an important role in neuronal differentiation of glioblastoma initiation cells, in addition to promoting their self-renewal ability $[5,28,29]$.

Further, in diffuse intrinsic pontine gliomas, recurrent somatic mutations of the activin receptor gene, ACVR1, were identified by combining whole-genome, whole-exome, methylome sequencing, and transcriptome [5,30]. These mutations result in constitutively activated proteins, which lead to phosphorylation of SMAD \& over expression of its downstream targets, inhibitor of DNA binding proteins $1 \& 2$ $[5,30]$. In pediatric midline high-grade astrocytomas, a gainof-function mutation in ACVR1 leads to hyper activation of bone morphogenetic protein (BMP)-ACVR1 pathway, resulting in increased phosphorylation of SMAD 1/5/8, and activation of BMP target genes [5,31]. In brain-stem gliomas, tumor-specific mutations in PPM1D were identified by targeted mutational analysis and exome sequencing [5,32]. Being high-frequency target of somatic mutation, PPM1D mutations enhance the cell's capacity to inhibit the activation of DNA damage response, making it a potential target in brain stem glioma treatment.

Another biomarker of great potential seems to be: the integrity of circulating cell-free (cfDNA) in plasma or serum, which appears to be a diagnostic and prognostic marker. During apoptosis and necrosis of cancer cells, cfDNA is released into the tumor microenvironment. cfDNAs are perfect targets for biopsies as they reflect disease progression and turnover in real time [33-35].

Data also indicate that miR-454-3p may serve as an exosomal biomarker and may be developed into a novel treatment for glioma patients [3].

Current therapies to abrogate tumor immunesuppression and unleash T-cell mediated killing of GBM include: (i) Inhibitors of immune checkpoints that are exploited by GBM to enhance tumor survival (therapeutic antibodies against CTLA-4 \& PD-1, which are over expressed in tumor infiltrating lymphocytes and against PD-L1, overexpressed on GBM cells \& tumor-infiltrating immune cells) (ii) Vaccination strategies against single or multiple tumorassociated peptides (iii) Infusion of autologous adoptive CAR T-cells primed against GBM-specific antigens \& (iv) Inhibition of immune-modulatory indole-amine 2,3-dioxygenase (IDO) pathway, which is also over-expressed in $\operatorname{GBM}[2,12,36]$.

\section{A dig into the process of biomarker discovery}

Over the past decade, proteomic analysis has undergone many technological advances allowing for the discovery of comprehensive proteomic profiles using glioma patient biopsies, biological fluids (plasma, serum, CSF, cyst fluid), glioma cell lines and animal models [4].

Biological Fluids: Body fluids are an excellent source of disease markers, as they are readily accessible and represent drainage products from involved tissues, including proteins secreted by normal, diseased, and dying cells such as inflammatory markers, immunoglobulins, cytokines, vasoactive compounds and mediators of cell signaling and/ or growth pathways [4,37]. The ability to follow such changes throughout the course of disease provides a unique opportunity to diagnose, prognose, predict and monitor the presence and progression of a specific pathologic process $[4,38,39]$.

Plasma/Serum: They have been valuable sample sources for human glioma biomarker research in the last many years [4,40-42] since it contains an intricate mixture of proteins, glucose, clotting factors, mineral ions, hormones $\& \mathrm{CO}_{2}$.

Cerebrospinal fluid: CSF, has of late emerged as a novel key source for biomarkers in glioma $[37,43,44]$. The fluid is composed of water, glucose, salts, metabolites, nucleic acids, peptides, proteins, and enzymes. Although CSF shares many properties with serum, such as the presence of albumin and immunoglobulin as its largest constituents and maintaining similar levels of salt and ions, several key features make CSF unique in its potential application in glioma proteomics.

Tissue: Direct analysis of tumor tissue represents a very good approach for glioma-specific protein biomarker discovery, as it is able to circumvent the highly dynamic and complex protein composition of biofluids $[4,45]$. Of course, not all markers identified by this approach will be detected in bodily fluids, as they may not be secreted proteins, their diffusion might be limited upon tumor cell death, or they may even be eliminated through phagocytosis. 
Antibody-driven techniques: Some of the current antibody-driven technologies for biomarker verification include western blot, dot blot, ELISA, protein chip, and peptide, protein, and tissue microarrays.

Western and Dot Blotting, ELISA, and Immunohistochemistry: Classical biochemistry techniques, including western and dot blots, ELISA, and IHC, are analytical methodologies used to detect target proteins by utilizing specific antibodies. For example, attractin was identified as a novel marker for malignant astrocytoma (WHO grades III and IV) in the CSF $[4,46]$.

Peptide, Protein and Tissue Arraying: Protein arrays, specifically antibody and aptamer arrays, represent one of the high-throughput techniques enabling simultaneous detection and monitoring of multiple proteins. In an antibody array, hundreds of antibodies directed at biomarkers of interest are immobilized on a solid surface and act as bait molecules that capture the solution phase analyte molecules from individual biospecimen protein mixes [4].

As an alternative proteomic technology, tissue microarrays (TMAs) play a key role in accelerating validation of identified protein biomarkers, with the potential for translating basic research findings into clinical applications [4].

There is no single molecular marker that can reveal the condition of all gliomas. Future studies on glioma biomarkers must focus on identification of specific associated molecules while improving the sensitivity of their detection along with the feasibility of the procedure. To better understand biological characteristics of glioma tumor, comprehensive analysis of associated multiple molecular markers should be performed. Despite all the advances, accurate and comprehensive diagnosis of glioma is still not feasible, thus keeping the challenge open for future studies [5].

\section{Methodologies for Data Acquisition and Processing}

Two major but complementary methodologies are widely utilized for downstream quantitative protein and peptide analysis in glioma proteomics research. Although gel-based protein profiling predominated in the early stages of proteomics, a mass spectrometry-based approach is emerging as the mainstream technology in proteomics today because it offers high level of resolution, facilitating subsequent analyses and improving confidence [4].

\section{Biomarker Verification}

Verification of biomarker panels is a mandatory step following its discovery. Low patient numbers and absence of standardized sample processing protocols may lead to discovery of false-positive proteins. Studies combining the information gained from genetic, transcriptomic and proteomic analyses of the same set of samples need to be performed for optimal selection of biomarkers [4]. Collectively, these are anticipated to eliminate false-positive targets and bridge the current gap between discovery, validation and translation of biomarkers into clinically relevant glioma targets in the near future [4].

\section{Conclusion}

Identifying the molecular \& pathogenetic characteristics of glioma regulation network may increase the precision of customized medication. But the exact role of immue biomarkers is still in its elementary stage. However, there is little doubt that the proteomic approach has the potential to identify novel diagnostic, prognostic, and therapeutic biomarkers. In fact, in the near future, improved proteomic profiling is anticipated to bring about a merger of biology, engineering, and informatics, with a profound impact on glioma research and treatment. Optimization of experimental design and validation in independent cohorts, improved multiplex proteomic methodologies and bioinformatics tools, and their integration with genetic and metabolomic profiling technologies promise to play critical roles in the post proteomics era of cancer diagnosis and treatment.

\section{Challenges}

Proteomic studies have already generated a sizable number of candidate diagnostic and prognostic markers in human brain cancer. Before adapting discovered biomarkers to the clinic, it is necessary to address some of the significant challenges that still remain in existing methodologies, hindering an in-depth, non-biased profiling of human glioma proteome. These include: reproducible detection, inter-technology reproducibility, lack of standards for proper study design \& collection/handling of bio-specimens, low sample numbers that result in inadequate statistical power.

\section{Conflicts of Interest}

Declared none.

\section{References}

1. Liang S, Shen G. Biomarkers of glioma. In: Garami M (ed). Molecular targets of CNS tumors. In Tech, 2011.

2. Buerki RA, Lukas RV. Serum immune-biomarkers in gliomas. Neuroimmunol Neuroinflammation. 2016; 3: 198-200. doi: 10.20517/2347-8659.2016.41

3. Shao N, Xue L, Wang R, Luo K, Zhi F, Lan Q. miR-454-3p is an exosomal biomarker and functions as a tumor suppressor in glioma. Mol Cancer Ther. 2019; 18(2): 459-469. doi: 10.1158/1535-7163.MCT-18-0725

4. Kalinina J, Peng J, Ritchie JC, Van Meir EG. Proteomics of gliomas: Initial biomarker discovery and evolution of technology. Neuro Oncol. 2011; 13(9): 926-942. doi: 10.1093/neuonc/nor078

5. Qi J, Yang $H$, Wang $X, T u ~ Y$. The progress in molecular biomarkers of gliomas. Cancer Transl Med. 2016; 2(4): 125-129. doi: 10.4103/23953977.189305

6. Riemenschneider MJ, Jeuken JW, Wesseling P, Reifenberger G. Molecular diagnostics of gliomas: state of the art. Acta Neuropathol. 2010; 120(5): 567-584. doi: 10.1007/s00401-010-0736-4

7. Tohma Y, Gratas C, Van Meir EG, et al. Necrogenesis and Fas/APO-1 (CD95) expression in primary (de novo) and secondary glioblastomas. J Neuropathol Exp Neurol. 1998; 57(3): 239-245. 
8. de Groot JF. High-grade gliomas. Continuum (Minneap Minn). 2015; 21: 332-344. doi: 10.1212/01.CON.0000464173.58262.d9

9. Wu CX, Lin GS, Lin ZX, Zhang JD, Liu SY, Zhou CF. Peritumoral edema shown by MRI predicts poor clinical outcome in glioblastoma. World J Surg Oncol. 2015; 13: 97. doi: 10.1186/s12957-015-0496-7

10. Stupp R, Mason WP, van den Bent MJ, et al. Radiotherapy plus concomitant and adjuvant temozolomide for glioblastoma. $N$ Engl $J$ Med. 2005; 352 (10): 987-996.

11. Bell C, Dowson N, Fay M, et al. Hypoxia imaging in gliomas with F-fluoromisonidazole PET: toward clinical translation. Semin Nucl Med. 2015; 45(2): 136-150. doi: 10.1053/j.semnuclmed.2014.10.001

12. Binder DC, Davis AA, Wainwright DA. Immunotherapy for cancer in the central nervous system: current and future directions. Oncoimmunology. 2015; 5(2): e1082027. doi: 10.1080/2162402X.2015.1082027

13. Lukas RV, Wainwright DA, Laterra JJ. Updates from the NeuroOncology Section of the 2015 American Neurological Association Annual Meeting. Future Oncol. 2016; 12(2): 143-147. doi: 10.2217/fon.15.299

14. Alexiou GA, Vartholomatos E, Zagorianakou P, Voulgaris S. Prognostic significance of neutrophil-to-lymphocyte ratio in glioblastoma. Neuroimmunol Neuroinflammation. 2014; 1: 131-134. doi: 10.4103/23478659.143666

15. Alexiou GA, Kallinteri A, Nita E, Zagorianakou P, Levidiotou S, Voulgaris S. Serum IgE levels in patients with intracranial tumors. Neuroimmunol Neuroinflammation. 2015; 2: 15-17. doi: 10.4103/2347-8659.149398

16. Bambury RM, Teo MY, Power DG, et al. The association of pre-treatment neutrophil to lymphocyte ratio with overall survival in patients with glioblastoma multiforme. J Neurooncol. 2013; 114(1): 149-154. doi: 10.1007/s11060-013-1164-9

17. Gorlia $\mathrm{T}$, van den Bent MJ, Hegi ME, et al. Nomograms for predicting survival of patients with newly diagnosed glioblastoma: prognostic factor analysis of EORTC and NCIC trial 26981-22981/CE.3. Lancet Oncol. 2008; 9(1): 29-38. doi: 10.1016/S1470-2045(07)70384-4

18. Gorlia $T$, Stupp $R$, Brandes $A A$, et al. New prognostic factors and calculators for outcome prediction in patients with recurrent glioblastoma: a pooled analysis of EORTC Brain Tumour Group phase I and II clinical trials. Eur J Cancer. 2012; 48: 1176-1184. doi: 10.1016/j. ejca.2012.02.004

19. Han $S$, Zhang $C$, Li $Q$, et al. Tumour-infiltrating CD4(+) and CD8(+) lymphocytes as predictors of clinical outcome in glioma. $\mathrm{Br} J$ Cancer. 2014; 110: 2560-2568. doi: 10.1038/bjc.2014.162

20. Han S, Liu Y, Li Q, Li Z, Hou H, Wu A. Pre-treatment neutrophiltolymphocyte ratio is associated with neutrophil and T-cell infiltration and predicts clinical outcome in patients with glioblastoma. BMC Cancer. 2015; 15: 617. doi: 10.1186/s12885-015-1629-7

21. Berghoff $A S$, Kiesel $B$, Widhalm $G$, et al. Programmed death ligand 1 expression and tumor-infiltrating lymphocytes in glioblastoma. Neuro Oncol. 2015; 17: 1064-1075. doi: 10.1093/neuonc/nou307

22. Zhai L, Dey M, Lauing KL, Gritsina G, et al. The kynurenine to tryptophan ratio as a prognostic tool for glioblastoma patients enrolling in immunotherapy. J Clin Neurosci. 2015; 22: 1964-1968. doi: 10.1016/j. jocn.2015.06.018

23. McCarthy BJ, Rankin K, Il'yasova D, et al. Assessment of type of allergy and antihistamine use in the development of glioma. Cancer Epidemiol Biomarkers Prev. 2011; 20: 370-378. doi: 10.1158/1055-9965.EPI-10-0948

24. Wigertz $A$, Lönn $S$, Schwartzbaum J, et al. Allergic conditions and brain tumor risk. Am J Epidemiol. 2007; 166: 941-950. doi: 10.1093/aje/kwm203

25. Huang RY, Neagu MR, Reardon DA, Wen PY. Pitfalls in the neuroimaging of glioblastoma in the era of antiangiogenic and immuno/targeted therapy - detecting illusive disease, defining response. Front Neurol. 2015; 6: 33. doi: 10.3389/fneur.2015.00033

26. Okada $H$, Weller $M$, Huang $R$, et al. Immunotherapy response assessment in neuro-oncology: a report of the RANO working group. Lancet Oncol. 2015; 16: e534-e542. doi: 10.1016/S1470-2045(15)00088-1
27. Ozawa T, Riester M, Cheng YK, et al. Most human non-GCIMP glioblastoma subtypes evolve from a common proneural-like precursor glioma. Cancer Cell. 2014; 26(2): 288-300. doi: 10.1016/j.ccr.2014.06.005

28. Annibali $D$, Whitfield JR, Favuzzi $E$, et al. Myc inhibition is effective against glioma and reveals a role for Myc in proficient mitosis. Nat Commun. 2014; 5: 4632. doi: 10.1038/ncomms5632

29. Zheng $\mathrm{H}$, Ying $\mathrm{H}$, Yan $\mathrm{H}$, et al. Pten and $\mathrm{p} 53$ converge on c-Myc to control differentiation, self-renewal, and transformation of normal and neoplastic stem cells in glioblastoma. Cold Spring Harb Symp Quant Biol. 2008; 73: 427-437. doi: 10.1101/sqb.2008.73.047

30. Wu G, Diaz AK, Paugh BS, et al. The genomic landscape of diffuse intrinsic pontine glioma and pediatric non-brainstem high-grade glioma. Nat Genet. 2014; 46(5): 444-450. doi: 10.1038/ng.2938

31. Buczkowicz P, Hoeman C, Rakopoulos $P$, et al. Genomic analysis of diffuse intrinsic pontine gliomas identifies three molecular subgroups and recurrent activating ACVR1 mutations. Nat Genet. 2014; 46(5): 451456. doi: $10.1038 /$ ng.2936

32. Fontebasso AM, Papillon-Cavanagh $\mathrm{S}$, Schwartzentruber $\mathrm{J}$, et al. Recurrent somatic mutations in ACVR1 in pediatric midline highgrade astrocytoma. Nat Genet. 2014; 46(5): 462-466. doi: 10.1038/ng.2950

33. Schwarzenbach $\mathrm{H}$, Hoon DS, Pantel K. Cell-free nucleic acids as biomarkers in cancer patients. Nat Rev Cancer. 2011; 11(6): 426-437. doi: 10.1038/nrc3066

34. Dawson SJ, Tsui DW, Murtaza M, et al. Analysis of circulating tumor DNA to monitor metastatic breast cancer. N Engl J Med. 2013; 368(13): 11991209. doi: 10.1056/NEJMoa1213261

35. Murtaza M, Dawson SJ, Tsui DW, et al. Non-invasive analysis of acquired resistance to cancer therapy by sequencing of plasma DNA. Nature. 2013; 497(7447): 108-112. doi: 10.1038/nature12065

36. Zhai $\mathrm{L}$, Lauing $\mathrm{KL}$, Chang $\mathrm{AL}$, et al. The role of IDO in brain tumor immunotherapy. J Neurooncol. 2015; 123: 395-403. doi: 10.1007/s11060014-1687-8

37. Khwaja FW, Van Meir EG. Proteomic discovery of biomarkers in the cerebrospinal fluid of brain tumor patients. In: Van Meir EG (ed). CNS cancer: Models, Prognostic Factors, Targets and Therapeutic Approaches. 1st edition. New York: Humana Press (Springer). 2009: 577-614.

38. D'Aguanno S, Del Boccio P, Bernardini $S$, et al. Electrophoretic separations of cerebrospinal fluid proteins in clinical investigations. Clin Chem Lab Med. 2007; 45(4): 437-449. doi: 10.1515/CCLM.2007.106

39. Kennedy S. Proteomic profiling from human samples: the body fluid alternative. Toxicol Lett. 2001; 120(1-3): 379-384.

40. Liu J, Zheng S, Yu JK, Zhang JM, Chen Z. Serum protein fingerprinting coupled with artificial neural network distinguishes glioma from healthy population or brain benign tumor. J Zhejiang Univ Sci B. 2005; 6(1): 4-10. doi: 10.1631/jzus.2005.B0004

41. Zhang $H$, Wu G, Tu $H$, Huang F. Discovery of serum biomarkers in astrocytoma by SELDI-TOF MS and proteinchip technology. J Neurooncol. 2007; 84(3): 315-323. doi: 10.1007/s11060-007-9376-5

42. Petrik V, Saadoun $S$, Loosemore A, et al. Serum alpha 2-HS glycoprotein predicts survival in patients with glioblastoma. Clin Chem. 2008; 54(4): 713-722. doi: 10.1373/clinchem.2007.096792

43. Van Meir EG, Hadjipanayis CG, Norden AD, Shu HK, Wen PY, Olson JJ. Exciting new advances in neuro-oncology: the avenue to a cure for malignant glioma. CA Cancer J Clin. 2010; 60(3): 166-193. doi: 10.3322/ caac.20069

44. Fountoulakis $\mathrm{M}$, Kossida $\mathrm{S}$. Proteomics-driven progress in neurodegeneration research. Electrophoresis. 2006; 27(8): 1556-1573. doi: 10.1002/elps.200500738

45. Niclou SP, Fack F, Rajcevic U. Glioma proteomics: status and perspectives. J Proteomics. 2010; 73(10): 1823-1838. doi: 10.1016/j. jprot.2010.03.007

46. Khwaja FW, Reed MS, Olson JJ, et al. Proteomic identification of biomarkers in the cerebrospinal fluid (CSF) of astrocytoma patients. J Proteome Res. 2007; 6(2): 559-570. doi: 10.1021/pr060240z 